Pathologe 2008 · [Suppl 2] 29:153-156 DOI 10.1007/s00292-008-1072-5

Online publiziert: 28. September 2008

(c) Springer Medizin Verlag 2008
K. Sotlar

Pathologisches Institut, Ludwig-Maximilians-Universität, München

\section{Die Infektion mit humanen Papillomaviren}

\section{Pathologie und Molekularpathologie}

Das Zervixkarzinom ist weltweit die zweithäufigste maligne Neoplasie der Frau, in jedem Jahr gibt es $500.000 \mathrm{Neu}-$ erkrankungen [18]. Jährlich sterben 275.00o Frauen an den Folgen einer Zervixkarzinomerkrankung, entsprechend 10\% aller krebsbedingten Todesfälle [18]. Bei mehr als 95\% aller Zervixkarzinome kann mit den heute zur Verfügung stehenden molekularbiologischen Methoden DNA bestimmter humaner Papillomaviren (HPV) nachgewiesen werden [17]. Zervixkarzinome entstehen auf dem Boden präinvasiver Vorstufen, der sog. zervikalen intraepithelialen Neoplasien (CIN). Diese sind im Rahmen der gesetzlich angebotenen Vorsorgeuntersuchungen identifizierbar und auch therapierbar.

Seit kurzem sind 2 prophylaktische Impfstoffe gegen die beiden häufigsten potenziell kanzerogenen HPV-Typen, HPV-16 und HPV-18, verfügbar. Eine prophylaktische Impfung wird seit November 2007 von der ständigen Impfkommission (STIKO) des Robert-KochInstituts empfohlen. Die Kosten für die Impfung werden von den gesetzlichen Krankenkassen getragen. HPV-Infektionen sind auch für die Entstehung von Genitalwarzen (Condylomata acuminata) sowie für einen erheblichen Anteil intraepithelialer Neoplasien der Vagina, Vulva und des Anus verantwortlich. Die Infektion erfolgt in aller Regel durch sexuellen Kontakt. Im Folgenden sollen die 4 als wesentlich angesehenen Schritte der zervikalen Karzinogenese [23] - HPV-Infektion, Viruspersistenz, Progression zur Krebsvorstufe und Invasion - dargestellt werden.

\section{HPV-Infektion}

Derzeit sind über 100 verschiedene HPVTypen bekannt, von denen etwa 40 die Haut und insbesondere die Schleimhäute des Anogenitaltrakts infizieren [30]. Die Übertragung erfolgt durch direkten Hautbzw. Schleimhautkontakt. Dabei scheint auch ein Virustransfer z. B. vom Introitus auf die Zervix ohne penetrierenden Geschlechtsverkehr möglich [32]. Erste HPV-Infektionen werden bereits kurz nach Aufnahme der sexuellen Aktivität gesehen. Verlaufsbeobachtungen bei 15bis 19-jährigen jungen Frauen zeigten eine kumulative Inzidenz von $60 \%$ innerhalb von 5 Jahren [33]. Die höchste HPVPrävalenz wird bei Frauen unter 25 Jahren gefunden. Sie nimmt in der Folge rapide $\mathrm{ab}$ und erreicht einen 2. Gipfel im Alter von 35-55 Jahren - im Bereich der höchsten Inzidenz des Zervixkarzinoms [6]. Die gleichzeitige Infektion mit mehreren verschiedenen HPV-Typen ist keine Seltenheit und wird in $20-30 \%$ der Fälle gefunden [8]. Das Lebenszeitrisiko einer HPVInfektion wird auf $80-90 \%$ geschätzt und wird stark durch die Anzahl der Sexualpartner beeinflusst [1]. Mehr als 90\% der Infektionen verlaufen transient [9].

Basierend auf dem Nachweis ihrer DNA in Zervixkarzinomen wird eine Gruppe von 15 HPV-Typen als potenziell karzinogen betrachtet und als sog. Highrisk-HPV- (HR-HPV-)Typen bezeichnet (- Tab. 1; $[17,25])$. Unter diesen ist HPV16 der mit Abstand am häufigsten nachgewiesene Typ und, zusammen mit HPV-18, für die Entstehung von etwa $70 \%$ der Zervixkarzinome und 50\% der CIN-3-Läsionen verantwortlich [25]. Demgegenüber sind die am häufigsten nachgewiesenen nichtkarzinogenen sog. Low-risk-HPVTypen (LR-HPV), HPV-6 und HPV-11, für die Entstehung von über 90\% der genitalen Kondylome verantwortlich [25].

Das HPV-Genom besteht aus nur 8 Genen, darunter 6 sog. "early genes“ (E6, E7, E1, E2, E4, E5) und 2 sog. „late genes" (L2, L1). Letztere kodieren für die beiden Untereinheiten des Viruskapsids. In bestimmten Regionen beider Gene bestehen große Sequenzhomologien zwischen den verschiedenen HPV-Typen, welche man sich für den Nachweis mittels Polymerase-Kettenreaktion (PCR) zu Nutze macht [20, 21]. Zu den „frühen Genen“ gehören die beiden viralen Onkogene E6 und E7. Beide besitzen mehrere zelluläre Zielmoleküle, wobei die wichtigsten Interaktionen in der E6-vermittelten Blockade der p53-induzierten Apoptose und in der E7-vermittelten Inhibition des pRB-induzierten Zellzyklusarrests liegen. Die Transkription der "frühen Gene“ wird über eine promotortragende sog. „long control region“ (LCR) reguliert. Diese besitzt u. a. Bindungsstellen für die viralen Proteine E1 und E2, welche unter normalen Umständen hierdurch die Expression der Onkogene E6 und E7 inhibieren [7].

Eine notwendige Voraussetzung zur Etablierung einer persistierenden Infektion ist die Infektion von Basalzellen des Plattenepithels, in welchen die Viren ihr Genom replizieren. Eine Infektion oberflächlicherer, nicht mehr teilungsfähiger Zelllagen würde dagegen unweigerlich $\mathrm{zu}$ einer nur transienten Infektion führen, da die Viren im Rahmen des normalen ZellTurnovers aus dem reifenden Plattenepithel „ausgewaschen“ würden. An die Ba- 


\begin{tabular}{|c|c|c|}
\hline \multirow[t]{2}{*}{ Genotyp } & Absolut & Kumulativ \\
\hline & (\%) & (\%) \\
\hline HPV-16 & 54,6 & 54,6 \\
\hline HPV-18 & 15,8 & 70,4 \\
\hline HPV-33 & 4,4 & 74,8 \\
\hline HPV-45 & 3,7 & 78,5 \\
\hline HPV-31 & 3,5 & 82,0 \\
\hline HPV-58 & 3,4 & 85,4 \\
\hline HPV-52 & 2,5 & 87,9 \\
\hline HPV-35 & 1,8 & 89,7 \\
\hline HPV-59 & 1,1 & 90,8 \\
\hline HPV-56 & 0,8 & 92,2 \\
\hline HPV-51 & 0,7 & 92,9 \\
\hline HPV-39 & 0,7 & 93,6 \\
\hline HPV-73 & 0,5 & 94,1 \\
\hline HPV-68 & 0,5 & 94,6 \\
\hline HPV-82 & 0,2 & 94,8 \\
\hline
\end{tabular}

salzellen gelangen Papillomaviren über kleinste Schleimhautverletzungen. Heparansulfat-Proteoglykane und a6-Integrin werden als mögliche Virusrezeptoren auf den Basalzellen vermutet $[5,24]$.

Latente HPV-Infektionen führen zu keinerlei morphologischer Auffälligkeiten, sondern sind ausschließlich molekularbiologisch nachzuweisen. Sie machen den weitaus größten Teil der HPVInfektionen aus. Molekularbiologisch sind latente Infektionen durch das Vorliegen rein episomaler Virus-DNA in den Basalzellen in niedriger Kopienzahl (etwa 20-100; daher meist nur mit sehr sensitiven Methoden nachweisbar, z. B. PCR) charakterisiert. Im Vordergrund steht die Expression von E1 und E2, mit entsprechender Suppression von E6 und E7 [4].

Über bislang nicht eindeutig aufgeklärte Mechanismen, möglicherweise eine Schwäche der T-Zell-vermittelten Immunabwehr, können auch transiente HPV-Infektionen in ein sog. „produktives" Stadium der Infektion übergehen. Erst jetzt ist die HPV-Infektion auch morphologisch nachweisbar: aufgrund gesteigerter Zellproliferation mit Plattenepithelhyperplasie, mit oder ohne diskrete Architekturstörungen [im Sinne einer geringgradigen zervikalen intraepithelialen Neoplasie (CIN 1)], oder durch das Auftreten von Koilozyten, als zytopathischer
Effekt einer gesteigerten Virusreplikation. Virus-DNA ist jetzt in hoher Kopienzahl über die gesamte Epithelbreite nachweisbar. Neben einer gesteigerten Expression des viralen E4-Proteins kommt es jetzt insbesondere auch zur Expression der Kapsidproteine L1 und L2. Das Virus ist nun in der Lage, seinen Lebenszyklus zu vollenden. In apikalen Zelllagen werden massenhaft infektiöse Viruspartikel produziert [15]. Für das therapeutische Management sollte ist in diesem Stadium berücksichtigt werden, dass die beschriebenen HPV-assoziierten morphologischen Veränderungen, bis hin zur CIN 1, eine Spontanregressionsrate von etwa $90 \%$ innerhalb von 36 Monaten besitzen [16].

\section{HPV-Persistenz}

Auch wenn die Definition der Persistenz aus virologischer Sicht problematisch sein kann und z. B. von der Sensitivität der verwendeten Nachweismethode (potenzieller Nachweis auch latenter Infektionen), der Länge der Intervalle zwischen 2 Tests (je kürzer, um so eher 2 -fach positiv) und dem Zeitpunkt des Eintritts in die Testung (kurz vor, während oder nach Infektion) abhängig ist [34], ist dies aus prognostischer Sicht im Rahmen einer HRHPV-Infektion bislang der mit Abstand zuverlässigste Risikofaktor für die Entstehung einer mäßig- oder schwergradigen zervikalen intraepithelialen Neoplasie (CIN 2/3). So nimmt mit fortschreitender Dauer der Infektion die Wahrscheinlichkeit eines spontanen Verschwindens des Virus ab, und es steigt gleichzeitig das Risiko der Entstehung einer Präkanzerose [19].

Eigene Untersuchungen deuten an, dass der einmalige Nachweis der transkriptionellen Aktivität der viralen Onkogene E6/E7 als Marker für eine Viruspersistenz dienen könnte [27, 28]. Die Ursache für eine Viruspersistenz ist unklar, hängt aber vermutlich ebenfalls mit einem Versagen der T-Zell-vermittelten Immunität $a b$, wie die deutlich höheren Raten persistierender und progredienter HR-HPV-Infektionen bei immunsupprimierten oder HIV-positiven HR-HPV-infizierten Menschen zeigen [29].

\section{Progression zur Krebsvorstufe}

Als eigentliche Präkanzerosen werden heute lediglich ein Teil der CIN-2-Läsionen sowie insbesondere die CIN-3-Läsionen angesehen. Dagegen gelten praktisch alle CIN-1-Läsionen und zumindest der durch LR-HPV induzierte Teil der CIN-2-Läsionen als Ausdruck zytopathologischer Effekte produktiver HPVInfektionen [23]. Die Vorstellung eines morphologischen Kontinuums von CIN 1 über $\mathrm{CIN}_{2}$ zu $\mathrm{CIN}_{3}$ ist damit praktisch verlassen worden. Als wesentlicher Risikofaktor für die Entstehung einer CIN2/3-Läsion wird in unterschiedlichen Studien die persistierende HR-HPV-Infektion, insbesondere mit HPV-16, identifiziert, teilweise mit einem absoluten Risiko von bis zu $40 \%$ nach 3 - bis 5 -jähriger Viruspersistenz [2]. In einer groß angelegten dänischen Studie zeigten Kjaer et al. nach 2-maligem HPV-16-Nachweis eine $\mathrm{CIN}-2 / 3$-Inzidenz von $50 \%$ innerhalb von 10 Jahren [13]. Insgesamt zeigen die verschiedenen HR-HPV-Typen ein unterschiedliches Maß an transformierendem Potenzial [11, 22]. Daneben spielen andere Faktoren, wie Nikotinabusus, Vielgeburtlichkeit und die Benutzung oraler Kontrazeptiva eine nur untergeordnete Bedeutung [23]. Bei Immuninkompetenten ist, wie auf dem Boden einer gesteigerten Viruspersistenz zu erwarten, auch das Progressionsrisiko gesteigert [29].

Molekularbiologisch zeigen echte Präkanzerosen eine stark gesteigerte, über die gesamte Epithelbreite nachweisbare Expression der viralen Onkogene E6 und E7. Die Expression von E4 und die Produktion von L1- und L2-Kapsidproteinen kommt zum Erliegen, und das Virus ist, im Sinne einer abortiven Infektion, nicht mehr in der Lage, seinen Lebenszyklus zu vollenden [15]. Unterstützt, möglicherweise aber auch entscheidend verursacht wird die Überexpression durch die in einem Teil der CIN 2/3, häufiger jedoch in Karzinomen nachweisbare Integration des $\mathrm{Vi}$ rusgenoms in das Wirtsgenom. Allerdings sind nur solche Virusintegrate relevant, bei denen es im Rahmen des Ringbruchs des zirkulären Virusgenoms zu einer Destruktion der viralen Gene E1 und/oder E2 kam. Hierdurch fällt der regulative Effekt durch deren Genprodukte auf die Transkription 
von E6/E7 weg. Darüber hinaus scheinen chimärische Virus-Host-Transkripte gegenüber den rein viralen E6/E7-Transkripten eine größere Stabilität zu besitzen [10]. Der Nachweis der Virusintegration ist allerdings technisch sehr aufwendig, besitzt eine relativ geringe Sensitivität und erscheint daher für Screeninguntersuchungen wenig geeignet [14].

\section{Invasion}

Die Hauptinzidenzraten des Zervixkarzinoms liegen zwischen dem 35 . und 55 . Lebensjahr und damit etwa 10-15 Jahre nach dem Hauptinzidenzalter für Präkanzerosen. Im Gegensatz zur De-novo-Ausbildung von $\mathrm{CIN}-2 / 3$-Läsionen, welche bereits innerhalb von 2-5 Jahren nach erstem Geschlechtsverkehr nachgewiesen wurden, beträgt der Zeitraum bis zur Entstehung eines invasiven Karzinoms, soweit aus obigen Zahlen und älteren Studien ableitbar, zwischen 5 und 15 Jahren. [12, 31]. Zeit, die benötigt wird, damit sich in den virustransformierten Zellen (epi)genetische Alterationen, die bislang nur unvollständig charakterisiert sind, ansammeln. In ihrer Summe oder in ihrer speziellen Kombination führen diese schließlich zur Ausbildung eines invasionsfähigen Phänotyps [26]. Risikofaktoren für die Invasion auf viraler Seite scheinen Infektionen mit HPV-16, HPV-18 und HPV-45 zu sein. Sie sind, im Vergleich zu den Präkanzerosen, in invasiven Karzinomen überrepräsentiert [3]. Da in Karzinomen fast immer integrierte Virusgenome als Quelle für eine kontinuierliche Überexpression der viralen Onkogene E6 und E7 gefunden werden, scheint auch die Integration einen Risikofaktor darzustellen.

\section{Korrespondenzadresse}

\section{Prof. Dr. K. Sotlar}

Pathologisches Institut, Ludwig-Maximilians-Universität Thalkirchner Straße 36, 80337 München Karl.Sotlar@Imu.de

Interessenkonflikt. Der korrespondierende Autor gibt an, dass kein Interessenkonflikt besteht.

\section{Literatur}

1. Baseman JG, Koutsky LA (2005) The epidemiology of human papillomavirus infections. J Clin Virol (Suppl 1) 32: S16-24

Pathologe 2008 • [Suppl 2] 29:153-156 DOI 10.1007/s00292-008-1072-5

(c) Springer Medizin Verlag 2008

\section{K. Sotlar}

Die Infektion mit humanen Papillomaviren. Pathologie und Molekularpathologie

\section{Zusammenfassung}

Das Zervixkarzinom ist mit jährlich 500.000 Neuerkrankungen die weltweit zweithäufigste maligne Neoplasie der Frau. Der Altersgipfel liegt zwischen dem 35. und 55. Lebensjahr. Persistierende Infektionen mit etwa 15 Genotypen humaner Papillomaviren (HPV) sind die notwendige Voraussetzung in der zervikalen Karzinogenese der Plattenepithelkarzinome, wie wohl auch der meisten Adenokarzinome. Das transformierende Potenzial der sog. High-risk-HPV- (HR-HPV-)Typen beruht auf der Interaktion der viralen Onkogenprodukte E6 und E7 mit den zellulären Tumorsuppressorproteinen p53 und pRB. Die resultierende Störung der Zellzykluskontrolle bereitet den Boden für die schließlich zur malignen Transformation führende Akkumulation von bis jetzt nur unvollständig bekannten genetischen und epigenetischen Alterationen. Präkanzerosen des Zervixkarzinoms, die sog. zervikalen intraepithelialen Neopla-

\section{Human papillomavirus infection. Pathology and molecular pathology}

\section{Abstract}

Cervical cancer is the second most frequent female malignoma worldwide and accounts for about 500,000 cases every year. The peak incidence lies between 35 and 55 years of age. Persistent infections with a group of 15 so-called high-risk human papillomaviruses (HR-HPV) are the cause of cervical carcinogenesis of squamous cell carcinomas and for most of the adenocarcinomas. The transforming potential of HR-HPVs is based on the interaction of viral oncogene products $\mathrm{E} 6$ and E7 with the cellular tumor suppressor proteins p53 and pRB. The resulting loss of cell cycle control sets the basis for additional, as yet only incompletely discovered, genetic and epigenetic changes, finally leading to invasive growth. Preneoplastic changes, cervical intraepithelial neoplasias, can be identi- sien (CIN), lassen sich zytologisch und histologisch identifizieren und sind therapierbar. Seit kurzem sind 2 prophylaktische Impfstoffe gegen die beiden häufigsten HR-HPV-Typen, HPV-16 und HPV-18, verfügbar. Eine Impfung wird seit November 2007 von der ständigen Impfkommission (STIKO) des Robert-KochInstituts für alle 12- bis 17-jährigen Mädchen empfohlen. Die Impfung mit 3 Dosen sollte vor dem ersten Geschlechtsverkehr abgeschlossen sein. HPV-Infektionen sind auch für die Entstehung von Genitalwarzen (Condylomata acuminata) sowie für einen erheblichen Anteil intraepithelialer Neoplasien der Vagina, Vulva und des Anus verantwortlich. Die Infektion erfolgt in aller Regel durch sexuellen Kontakt.

Schlüsselwörter HPV · CIN · Zervixkarzinom · Karzinogenese · Impfung fied morphologically, thus allowing for therapeutic interventions. Since November 2007, the Ständige Impfkommission, the German standing committee on immunizations, has recommended the prophylactic use of vaccines against the two most frequent HR-HPV genotypes, HPV-16 and HPV-18, in women aged 12-17 years before first sexual intercourse. In addition to cervical cancer, HPV infections are responsible for the development of genital warts (condyloma) and a number of vaginal, vulvar, and anal intraepithelial neoplasias. HPV infections are usually transmitted sexually.

\section{Keywords}

HPV - CIN · Cervical cancer - Carcinogenesis .

Vaccination 
2. Castle PE, Solomon D, Schiffman M, Wheeler CM (2005) Human papillomavirus type 16 infections and 2-year absolute risk of cervical precancer in women with equivocal or mild cytologic abnormalities. J Natl Cancer Inst 97: 1066-1071

3. Clifford GM, Smith JS, Aguado T, Franceschi S (2003) Comparison of HPV type distribution in high-grade cervical lesions and cervical cancer: a meta-analysis. Br J Cancer 89: 101-105

4. Doorbar J (2006) Molecular biology of human papillomavirus infection and cervical cancer. Clin Sci (Lond) 110:525-541

5. Evander M, Frazer IH, Payne E et al. (1997) Identification of the alpha6 integrin as a candidate receptor for papillomaviruses. J Virol 71: 2449-2456

6. Franceschi S, Herrero R, Clifford GM et al. (2006) Variations in the age-specific curves of human papillomavirus prevalence in women worldwide. Int J Cancer 119: 2677-2684

7. Hebner CM, Laimins LA (2006) Human papillomaviruses: basic mechanisms of pathogenesis and oncogenicity. Rev Med Virol 16: 83-97

8. Herrero R, Castle PE, Schiffman M et al. (2005) Epidemiologic profile of type-specific human papillomavirus infection and cervical neoplasia in Guanacaste, Costa Rica. J Infect Dis 191: 1796-1807

9. Ho GY, Bierman R, Beardsley L et al. (1998) Natural history of cervicovaginal papillomavirus infection in young women. N Engl J Med 338: 423-428

10. Jeon S, Lambert PF (1995) Integration of human papillomavirus type 16 DNA into the human genome leads to increased stability of E6 and E7 mRNAs: implications for cervical carcinogenesis. Proc Natl Acad Sci U S A 92: 1654-1658

11. Khan MJ, Castle PE, Lorincz AT et al. (2005) The elevated 10-year risk of cervical precancer and cancer in women with human papillomavirus (HPV) type 16 or 18 and the possible utility of type-specific HPV testing in clinical practice. J Natl Cancer Inst 97: 1072-1079

12. Kinlen LJ, Spriggs Al (1978) Women with positive cervical smears but without surgical intervention. A follow-up study. Lancet 2: 463-465

13. Kjaer S, Høgdall E, Frederiksen K et al. (2006) The absolute risk of cervical abnormalities in high-risk human papillomavirus-positive, cytologically normal women over a 10-year period. Cancer Res 66 : 10630-10636

14. Klaes R, Woerner SM, Ridder R et al. (1999) Detection of high-risk cervical intraepithelial neoplasia and cervical cancer by amplification of transcripts derived from integrated papillomavirus oncogenes. Cancer Res 59: 6132-6136

15. Middleton K, Peh W, Southern S et al. (2003) Organization of human papillomavirus productive cycle during neoplastic progression provides a basis for selection of diagnostic markers. J Virol 77: 1018610201

16. Moscicki AB, Shiboski S, Hills NK et al. (2004) Regression of low-grade squamous intra-epithelial lesions in young women. Lancet 364: 1678-1683

17. Muñoz N, Bosch FX, de Sanjosé S et al. and International Agency for Research on Cancer Multicenter Cervical Cancer Study Group (2003) Epidemiologic classification of human papillomavirus types associated with cervical cancer. N Engl J Med 348: 518-527

18. Parkin DM, Bray F, Ferlay J, Pisani P (2005) Global cancer statistics, 2002. CA Cancer J Clin 55: 74-108

19. Plummer M, Schiffman M, Castle PE et al. and ALTS Group (2007) A 2-year prospective study of human papillomavirus persistence among women with a cytological diagnosis of atypical squamous cells of undetermined significance or low-grade squamous intraepithelial lesion. J Infect Dis 195: 1582-1589
20. Resnick RM, Cornelissen MT, Wright DK et al. (1990) Detection and typing of human papillomavirus in archival cervical cancer specimens by DNA amplification with consensus primers. J Natl Cancer Inst 82: $1477-1484$

21. de Roda Husman AM, Walboomers JM, van den Brule AJ et al. (1995) The use of general primers GP5 and GP6 elongated at their $3^{\prime}$ ends with adjacent highly conserved sequences improves human papillomavirus detection by PCR. J Gen Virol 76: 1057-1062

22. Schiffman M, Herrero R, Desalle R et al. (2005) The carcinogenicity of human papillomavirus types reflects viral evolution. Virology 337: 76-84

23. Schiffman M, Castle PE, Jeronimo J et al. (2007) Human papillomavirus and cervical cancer. Lancet 370: 890-907

24. Shafti-Keramat $S$, Handisurya A, Kriehuber E et al. (2003) Different heparan sulfate proteoglycans serve as cellular receptors for human papillomaviruses. JVirol 77: 13125-13135

25. Smith JS, Lindsay L, Hoots B et al. (2007) Human papillomavirus type distribution in invasive cervical cancer and high-grade cervical lesions: a meta-analysis update. Int J Cancer 121: 621-632

26. Snijders PJ, Steenbergen RD, Heideman DA, Meijer CJ (2006) HPV-mediated cervical carcinogenesis: concepts and clinical implications. J Pathol 208: 152-164

27. Sotlar K, Stubner A, Diemer D et al. (2004) Detection of high-risk human papillomavirus E6 and E7 oncogene transcripts in cervical scrapes by nested RT-polymerase chain reaction. J Med Virol 74: 107116

28. Sotlar K, Diemer D, Stubner A et al. (2005) Detection of high-risk human papillomavirus (HPV) E6 and E7 oncogene transcripts increases the specificity of the detection of a cervical intraepithelial neoplasia (CIN). Verh Dtsch Ges Pathol 89: 195-200

29. Strickler HD, Burk RD, Fazzari M et al. (2005) Natural history and possible reactivation of human papillomavirus in human immunodeficiency viruspositive women. J Natl Cancer Inst 97: 577-586

30. de Villiers EM, Fauquet C, Broker TR et al. (2004) Classification of papillomaviruses. Virology 324 : 17-27

31. Wallin KL, Wiklund F, Angström T et al. (1999) Typespecific persistence of human papillomavirus DNA before the development of invasive cervical cancer. N Engl J Med 341: 1633-1638

32. Winer RL, Lee SK, Hughes JP et al. (2003) Genital human papillomavirus infection: incidence and risk factors in a cohort of female university students. Am J Epidemiol 157: 218-226

33. Woodman CB, Collins S, Winter H et al. (2001) Natural history of cervical human papillomavirus infection in young women: a longitudinal cohort study. Lancet 357: 1831-1836

34. Woodman CB, Collins SI, Young LS (2007) The natural history of cervical HPV infection: unresolved issues. Nat Rev Cancer 7: 11-22 